\title{
Correction to: Data Engineering and Intelligent Computing
}

Vikrant Bhateja, Suresh Chandra Satapathy, Carlos M. Travieso-González, and V. N. Manjunath Aradhya

\section{Correction to:}

V. Bhateja et al. (eds.),

Data Engineering and Intelligent Computing, Advances

in Intelligent Systems and Computing 1, https://doi.org/10.1007/978-981-16-0171-2

The original version of the book was inadvertently published with incorrect volume number, and this has been corrected as 1407 . This has now been amended throughout the book to the correct volume number. 